Mens

revue d'histoire intellectuelle de l'Amérique française

MENS

\title{
Le libéralisme dans la pensée religieuse de $\mathrm{M}^{\mathrm{gr}}$ Bruchési
}

\section{Francis Primeau}

Volume 7, numéro 2, printemps 2007

URI : https://id.erudit.org/iderudit/1024125ar

DOI : https://doi.org/10.7202/1024125ar

Aller au sommaire du numéro

Éditeur(s)

Centre de recherche en civilisation canadienne-française

ISSN

1492-8647 (imprimé)

1927-9299 (numérique)

Découvrir la revue

Citer cet article

Primeau, F. (2007). Le libéralisme dans la pensée religieuse de $\mathrm{M}^{\mathrm{gr}}$ Bruchési. Mens, 7(2), 241-277. https://doi.org/10.7202/1024125ar

\section{Résumé de l'article}

Cet article aborde le rapport entre le libéralisme et le catholicisme par l'étude de la pensée de $\mathrm{M}^{\mathrm{gr}}$ Paul-Napoléon Bruchési. Il révèle que, dans la pratique, nombreux sont les points de rencontre entre le catholicisme québécois et le libéralisme historique, conservateur, de tradition britannique. Certes, des tensions ont toujours existé tant au niveau théorique que pratique entre les deux idéologies puisqu'elles sont basées sur deux conceptions différentes du monde, la première véhiculant une vision organique de la société reposant sur un principe transcendant, la seconde, une vision atomisée relevant d'un immanentisme. Toutefois, ces tensions n'ont pas empêché les accommodements pratiques entre elles dans le but de maintenir le nouvel ordre social en place. Néanmoins, un ennemi est identifié, un combat est mené par les défenseurs du catholicisme. Cet ennemi n’est pas la modernité ou le libéralisme dans son ensemble, mais un élément présent dans la synthèse libérale : l'individualisme anthropocentrique et matérialiste. Chez $\mathrm{M}^{\mathrm{gr}}$ Bruchési, c'est principalement de ce point que découle tous les autres problèmes associés à l'émergence du monde moderne.
Ce document est protégé par la loi sur le droit d'auteur. L'utilisation des services d'Érudit (y compris la reproduction) est assujettie à sa politique d'utilisation que vous pouvez consulter en ligne.

https://apropos.erudit.org/fr/usagers/politique-dutilisation/ 


\title{
LE LIBÉRALISME DANS LA PENSÉE RELIGIEUSE DE $M^{\mathrm{GR}}$ BRUCHÉSI
}

\author{
Francis Primeau \\ Département d'histoire \\ Université Laval
}

\section{Résumé}

Cet article aborde le rapport entre le libéralisme et le catholicisme par l'étude de la pensée de $\mathrm{M}^{\mathrm{gr}}$ Paul-Napoléon Bruchési. Il révèle que, dans la pratique, nombreux sont les points de rencontre entre le catholicisme québécois et le libéralisme historique, conservateur, de tradition britannique. Certes, des tensions ont toujours existé tant au niveau théorique que pratique entre les deux idéologies puisqu'elles sont basées sur deux conceptions différentes du monde, la première véhiculant une vision organique de la société reposant sur un principe transcendant, la seconde, une vision atomisée relevant d'un immanentisme. Toutefois, ces tensions n'ont pas empêché les accommodements pratiques entre elles dans le but de maintenir le nouvel ordre social en place. Néanmoins, un ennemi est identifié, un combat est mené par les défenseurs du catholicisme. Cet ennemi n'est pas la modernité ou le libéralisme dans son ensemble, mais un élément présent dans la synthèse libérale : l'individualisme anthropocentrique et matérialiste. Chez $\mathrm{M}^{\mathrm{gr}}$ Bruchési, c'est principalement de ce point que découle tous les autres problèmes associés à l'émergence du monde moderne.

\section{Abstract}

This article explores the relationship between liberalism and Catholicism through the thought and writing of Msgr. Paul-Napoléon Bruchési. It reveals that, in practice, some degree of overlap existed between French Canadian Catholicism and British-style liberalism, a doctrine which possessed a historically conservative 
sensibility. As a result of their distinct worldviews, tensions did exist at both the practical and theoretical levels between liberalism and Catholicism. Friction was bound to arise between an organic vision of society based on the principle of transcendence and an atomized vision of society resting on immanentism. However, these tensions did not preclude practical accommodations, in an effort to uphold. the new social order, between liberalism and Catholicism. Msgr. Bruchési nevertheless identified a noxious doctrine within liberalism: anthropocentric and materialistic individualism. For the archbishop, the problems of the modern world flowed from this fallacy.

Quatrième évêque et deuxième archevêque de Montréal, $\mathrm{M}^{\mathrm{gr}}$ Paul-Napoléon Bruchési est une figure marquante de l'Église catholique dans l'histoire du Québec. Reconnu pour son intelligence vive et pour son éloquence, c'est sous son épiscopat que l'Oratoire Saint-Joseph (1904) est fondé et que se déroule à Montréal le vingtième Congrès eucharistique international (1910). C'est encore sous son leadership que l'Université de Montréal obtient son autonomie de l'Université Laval (1919) ${ }^{1}$. Grand intellectuel, formé dans les meilleurs séminaires, $\mathrm{M}^{\mathrm{gr}}$ Bruchési est promis dès sa jeunesse aux plus hautes responsabilités ecclésiastiques. Après des études à Rome où il obtient un doctorat en théologie et un autre en droit canonique, il reçoit l'ordination sacerdotale en 1878, alors qu'il n'a que vingt-quatre ans ${ }^{2}$. De retour à Montréal en 1879, il est d'abord secrétaire particulier de l'évêque de Montréal, $\mathrm{M}^{\mathrm{gr}}$ Édouard-Charles Fabre. Puis, de 1880 à 1884, il enseigne le dogme à l'Université Laval de Québec. En 1885, il est successivement vicaire aux paroisses Saint-Joseph et Sainte-Brigide et curé de la cathédrale de Montréal de 1891 à 1897. C'est en 1897, six mois après la mort de $\mathrm{M}^{\mathrm{gr}}$ Fabre, que le chanoine Bruchési est nommé archevêque de Montréal ${ }^{3}$. En poste officiellement de 1897 à 1939, $\mathrm{M}^{\mathrm{gr}}$ Bruchési a une vie épiscopale très active. Mais, en 1919, un mal étrange, caractérisé par un mélange de dépression physique et d'angoisse 
morale, lui enlève progressivement toute initiative et toute capacité de décision. Atteint par la maladie et vivant en réclusion, $\mathrm{M}^{\mathrm{gr}}$ Bruchési est remplacé dans l'administration du diocèse par $\mathrm{M}^{\mathrm{gr}}$ Gauthier en 1921. Ce dernier est nommé coadjuteur en $1923^{4}$.

Conservateur comme tous les évêques canadiens-français du début du siècle, mais ayant sous sa responsabilité la ville québécoise la plus moderne et cosmopolite, $\mathrm{M}^{\mathrm{gr}}$ Bruchési a surtout retenu l'attention des historiens pour sa lutte menée contre l'immoralité ${ }^{5}$. Dans une période trouble pour l'Église au Québec, alors que la modernité envahissait graduellement l'univers idéologique de la province, $\mathrm{M}^{\mathrm{gt}}$ Bruchési fait figure exemplaire dans la résistance cléricale aux changements causés par l'industrialisation et l'urbanisation. Le portrait de l'archevêque de Montréal est donc celui d'un acteur historique agissant à contre-courant de l'idéologie libérale en émergence au début du siècle, retardant ainsi la marche du Québec vers la modernité 6 .

Il est vrai qu'à la suite d'une lecture sommaire des écrits de l'archevêque, il est facile de conclure rapidement au refus et au rejet en bloc de l'individualisme, ainsi que de l'idéologie libérale qui en est le corollaire. À plusieurs reprises dans sa correspondance, $\mathrm{M}^{\mathrm{gr}}$ Bruchési fait allusion aux « erreurs philosophiques » ou aux «mauvaises doctrines » qui sont à la source de tous les malheurs liés au monde moderne. Tout nous porte à croire que c'est bien, en premier lieu, au libéralisme ainsi qu'à la nouvelle conception de la vie dont il est porteur que s'oppose formellement $\mathrm{M}^{\mathrm{gr}}$ Bruchési. Mais une analyse en profondeur des notions fondamentales libérales présentes dans les écrits de l'archevêque, ainsi que leur utilisation, forcent à constater que l'hostilité et la crainte manifestées par l'archevêque semblent viser moins la façon dont le libéralisme s'est réalisé dans la réalité historique que ce vers quoi il tend, 
c'est-à-dire l'évacuation du caractère transcendant de l'existence humaine et l'horizontalité de toute la vie. Dans sa correspondance, ainsi que dans ses mandements, les foudres de $\mathrm{M}^{\mathrm{gr}}$ Bruchési sont dirigées principalement contre un libéralisme doctrinaire ou philosophique véhiculant des idéaux sur l'existence humaine faisant table rase du caractère sacré et éternel de cette dernière. Dans ses écrits, $M^{\text {gr }}$ Bruchési annonce essentiellement le retour du paganisme des sociétés antiques et la présence d'un " Mal », d'un «Ennemi », qui sape la transmission des saines traditions. Pourtant, une acculturation partielle de sa pensée s'effectue par l'intégration et l'ajustement de certaines notions fondamentales inhérentes au libéralisme. Surtout, des ententes pratiques, souvent tacites, semblent se nouer avec un libéralisme historique qui s'éloigne de l'idéal projeté au sein de la doctrine libérale : un libéralisme de tradition britannique sans crispation anticléricale.

Pour appuyer notre réflexion, cette étude propose l'analyse des différentes notions fondamentales de la synthèse libérale (liberté, égalité, propriété et individualisme) et du sens que leur prête l'archevêque pour évaluer la part de rejet et d'intégration du libéralisme dans la pensée de $\mathrm{M}^{\mathrm{gr}}$ Bruchési. Cette analyse s'effectue principalement à partir de trois lettres pastorales. L'affaiblissement de l'esprit chrétien et le goût des plaisirs du monde, publiée le 16 décembre 1901, est l'une des plus longues lettres pastorales que $\mathrm{M}^{\mathrm{gr}}$ Bruchési a écrites. Son intention première réside dans une volonté de prévenir la population contre le danger que constitue pour la jeunesse et la famille la présence accrue de théâtres étrangers dans la ville de Montréal. Ce faisant, $\mathrm{M}^{\mathrm{gr}}$ Bruchési se prononce clairement sur ce qu'il perçoit comme étant la source de tous les malheurs qui menacent la famille, ainsi que la société canadiennefrançaise, à l'aube du $\mathrm{XX}^{\mathrm{e}}$ siècle. Dans la lettre pastorale Sur la question ouvrière, publiée le 23 avril 1903, M ${ }^{\mathrm{gr}}$ Bruchési cher- 
che d'abord à prévenir le danger de grève qui pèse sur la ville de Montréal et à calmer les ardeurs des ouvriers et des patrons sujets à avoir recours à la violence dans le but de promouvoir leurs intérêts respectifs. Il expose par le fait même la menace des intérêts égoïstes sur l'ordre social instauré par Dieu sur terre. Enfin, la lettre pastorale Sur la justice, parue le 4 novembre 1915, se veut un appel aux devoirs chrétiens pour l'ensemble de la société canadienne-française. Dans cette lettre pastorale, $\mathrm{M}^{\mathrm{gr}}$ Bruchési fait part de ses inquiétudes sur l'émiettement de la vertu de justice face à la vanité et à l'égoisme grandissants dans nos sociétés. Ce faisant, il réitère l'importance du respect de l'ordre comme source de justice et de paix sociale.

\section{La liberté}

Dans la synthèse libérale, la notion fondamentale de liberté se présente comme une prérogative de la nature humaine, un droit naturel qui permet à l'homme de disposer de sa personne et de ses biens comme il lui convient. L'homme est un être raisonnable capable de définir lui-même sa conduite et le chemin à suivre pour atteindre le bonheur? ${ }^{7}$. Ainsi présentée, la notion de liberté n'entre pas en contradiction avec la pensée de l'archevêque. En effet, pour $\mathrm{M}^{\mathrm{gr}}$ Bruchési, l'homme est bel et bien un être libre, Dieu ayant lui-même accordé cette liberté. L'homme est également un être doté de raison, faculté qui le rend apte à prendre des décisions pour sa personne. Les tensions entre le libéralisme en général et le catholicisme de $\mathrm{M}^{\mathrm{gr}}$ Bruchési ne trouvent pas leurs racines dans la notion de liberté individuelle comme telle, c'est-àdire comme un droit fondamental donné à l'être humain raisonnable afin qu'il puisse disposer lui-même de sa personne. Elles surgissent, d'une part, de la définition de la limite à imposer à cette liberté et, d'autre part, d'un doute présent chez 
l'archevêque à propos de la capacité pratique de l'homme moderne d'avoir recours à sa raison afin d'utiliser convenablement la liberté qui lui a été donnée : «Aussi, n'avons-nous nullement l'intention d'entraver cette juste liberté, dont Dieu lui-même a laissé l'exercice aux chrétiens qui ne sont pas tenus, par une vocation spéciale, à l'observance de tous les conseils évangéliques. Mais, en notre qualité de gardien de la foi et des mœurs, nous voudrions établir clairement, à vos yeux, la ligne de démarcation entre l'usage et l'abus gravement périlleux ou coupable des choses de ce monde ${ }^{8}$.»

Commençons d'abord par l'explication de l'idée de limite. Au sein du libéralisme, la liberté accordée à l'homme doit trouver une limite dans la liberté des autres. La liberté d'un individu se voit donc contrebalancée par le respect de l'ordre et de l'harmonie sociale imposé par la vie en collectivité. Ainsi, tous les hommes sont libres d'assouvir leurs désirs pourvu qu'ils ne contreviennent pas à la loi. La limite à la liberté de l'homme est essentiellement une limite imposée aux gestes et aux actions d'un individu qui risquent de perturber l'harmonie collective. Pour $\mathrm{M}^{\mathrm{gr}}$ Bruchési, pareille liberté laissée à l'homme ne peut être que synonyme d'anarchie et de dégénérescence sociale. Certes, l'homme libre vivant en société se doit de respecter l'ordre ainsi que la loi humaine qui s'y rattache. D'où d'ailleurs l'importance de l'État ou de l'autorité temporelle qui doit veiller à l'application et au respect de la loi humaine. Mais cela ne s'arrête pas là. L'homme, étant plus qu'une machine naturelle, est avant tout une créature de Dieu. Répondant aux lois humaines et positives qui régissent la société dans laquelle il vit, l'homme doit également respecter la loi éternelle, la grande loi surnaturelle qui régit son existence même. La limite imposée à la liberté individuelle est ainsi plus sévère selon une conception thomiste qui, contrairement à la philosophie du libéralisme, n'évacue pas le carac- 
tère métaphysique, transcendant et surnaturel attaché à la personne humaine.

Par conséquent, la liberté de l'homme ne constitue pas seulement un dérivé de la nature des choses ou une prérogative de la nature humaine. La liberté est un droit accordé à l'homme par Dieu, un droit dont l'exercice est indissociable de la capacité à choisir le Bien. Selon Bruchési, l'homme est revêtu d'une dignité de nature', une dignité dont il ne peut se détacher. Dieu ayant créé l'homme à son image, ce dernier est demeuré très proche de son créateur, d'une part par sa ressemblance au Créateur et, d'autre part, par sa participation aux desseins voulus par Dieu. Il s'agit donc de l'image de Dieu dans l'homme qui fonde la dignité, mais également la liberté de la personne humaine. La dignité naturelle de l'homme se devant d'être promue et respectée, il n'est permis à personne de la violer. Surtout, il n'est pas permis à l'homme de déroger à la dignité de sa nature, car la liberté qui lui est accordée par Dieu ne se constitue pas seulement de droits dont il a la libre disposition, mais également de devoirs envers Dieu qu'il doit remplir ${ }^{10}$. L'homme se doit donc d'agir selon la dignité de sa nature, de vivre de façon raisonnable, donc de se conformer librement aux desseins de son Créateur afin de ne pas obscurcir l'image divine qui est en lui.

À ce respect de la dignité humaine, fondement de la liberté de l'homme, $\mathrm{M}^{\mathrm{gr}}$ Bruchési ajoute la finalité divine de l'homme ainsi que l'inévitabilité du Jugement dernier. L'homme, créature mortelle, ne peut échapper à sa mort et au fait qu'un jour il devra rendre des comptes à son Créateur. $\mathrm{Ne}$ pouvant s'affranchir de cette réalité, il se doit de vivre de façon vertueuse et honorable pour s'assurer une place dans le royaume des cieux. Or la vertu requiert la maitrise des passions, issues du corps, qui doivent être subordonnées à la raison, liée à l'âme. Étant doté d'une raison, d'une âme, la fa- 
culté supérieure, l'homme doit ordonner ses passions, dominer son corps et son appétit sensitif en vue de sa fin ultime : la béatitude. Il s'agit là d'un des principes maîtres de la pensée thomiste de $\mathrm{M}^{\mathrm{gr}}$ Bruchési : la limite à l'exercice de la liberté doit être imposée de façon personnelle et doit se dresser à l'intérieur de chaque individu qui, lui-même, par une sorte de processus d'autorégulation, doit assurer la domination de sa raison sur ses passions et, par conséquent, garantir le juste exercice de sa liberté. La raison et la liberté fondent ainsi l'agir moral.

$\mathrm{M}^{\mathrm{gr}}$ Bruchési ne s'oppose donc pas formellement à la notion de liberté individuelle comme droit fondamental. Ce qu'il dénonce au sein du libéralisme, c'est l'éloge et la promotion d'une mauvaise liberté, une liberté qui semble rejeter toute autorité, une liberté qui vise l'assouvissement de toutes les passions du corps et qui pousse l'homme à se rapprocher plus de l'animal que de l'être divin. Il s'élève aussi contre une liberté moderne trop permissive, qui ne tient pas compte de la finalité divine de l'homme et qui tend à se libérer d'un esprit chrétien marqué par les valeurs d'humilité, de force, de tempérance et d'honneur ${ }^{11}$ : «En effet, le mal dont souffrent le plus les âmes aujourd'hui est sans contredit le mal de l'indépendance et de l'insubordination ${ }^{12}$. Il se dresse contre une liberté qui encourage l'excès, la démesure et qui flatte l'orgueil, pour défendre ce qu'il nomme «l'exercice honnête de la liberté ». Selon lui, cette liberté doit être équilibrée par l'accomplissement des devoirs chrétiens ainsi que par le respect des saines traditions. Par analogie, tout comme l'État libéral se fait le gardien de la loi humaine, $\mathrm{M}^{\mathrm{gr}}$ Bruchési perçoit l'Église comme la gardienne de la loi éternelle. Sa mission est d'offrir un encadrement qui permet de conseiller les fidèles afin qu'ils fassent des choix raisonnables qui les mèneront sur le chemin du salut éternel. 
Ainsi, en principe, l'encadrement des fidèles et l'autorité de l'Église n'ont pas pour but de contraindre l'individu sur le chemin à suivre, mais d'éclairer sa raison. $\mathrm{M}^{\mathrm{gr}}$ Bruchési accepte donc une certaine subjectivité dans la mesure où l'Église n'intervient que pour dicter la limite à ne pas dépasser. Toutefois, face à l'adversité et à l'abondance offerte par la société industrielle, $\mathrm{M}^{\mathrm{gr}}$ Bruchési doute des capacités de l'homme à effectuer les bons choix. Surtout, face à la propagation d'une conception essentiellement matérialiste de la vie qui accompagne l'urbanisation et l'industrialisation, $\mathrm{M}^{\mathrm{gr}}$ Bruchési ne peut qu'intervenir de plus en plus directement dans la vie des fidèles afin de limiter la « recherche effrénée et inépuisable des biens de la terre ${ }^{13} »$. En effet, dans la notion de liberté se trouve la question du choix, la question de la subjectivité. Face à toutes les tentations qu'offre le monde moderne, l'homme est-il assez raisonnable pour effectuer les bons choix ? En définitive, n'est-ce pas là, dans l'émergence d'une philosophie matérialiste et anthropocentrique, libérée du principe transcendant, que se trouve la véritable source du problème moderne pour $\mathrm{M}^{\mathrm{gr}}$ Bruchési ?

\section{L'égalité}

Dans le libéralisme, la notion d'égalité signifie une égalité dans le droit à la liberté, une égalité des chances et non la négation des différences et du mérite individuel. À l'état naturel, les hommes sont tous, à leur naissance, non seulement libres, mais égaux théoriquement. En raison des qualités personnelles acquises ou développées, certains auront par la suite plus de facilité à survivre et se distingueront des autres. Une inégalité peut donc survenir entre les individus libres, une inégalité qui n'est pas nécessairement imposée par des contraintes extérieures, mais qui découle du mérite individuel de chacun. En société, cette notion d'égalité se traduit par une 
égalité dans les droits et dans les chances de s'enrichir et d'acquérir des biens. À la naissance, les individus sont égaux entre eux dans leur capacité théorique à travailler et à exercer leur liberté. Les inégalités surgissent donc théoriquement en fonction de la réussite personnelle dans l'exercice de la liberté individuelle inhérente à chaque individu. Il ne s'agit en aucun cas d'une égalité des conditions qui est défendue au sein de l'idéologie libérale, mais bien d'une égalité dans les droits et les possibilités d'accumuler des richesses. Subordonnée à la liberté ainsi qu'à la propriété, l'égalité moderne supporte donc les inégalités de conditions perçues comme étant immanentes, dans la nature même des choses.

Bien qu'elle demeure une valeur subordonnée au sein de la synthèse libérale, l'égalité est nécessaire au libéralisme afin de réguler les relations entre les différents individus qui, dans le libre exercice de leur liberté, entrent en conflit les uns avec les autres. Tous les individus étant théoriquement égaux dans leur droit à la liberté, chacun doit par conséquent respecter la liberté de l'autre dans l'exercice de sa propre liberté. L'harmonie est donc assurée par une égalité de droit qui ellemême est assurée par la loi. L'ordre libéral repose donc théoriquement sur une égalité entre les individus libres et sur le respect de leurs droits dans la rivalité théorique pour l'acquisition de la richesse et de la propriété. Étant égaux en droits, les individus demeurent cependant, par nature, socialement inégaux. L'ordre libéral repose en fait sur une inégalité des conditions défendues au nom de l'individualisme et de la propriété privée. Perçue comme naturelle et comme le reflet des volontés individuelles, l'inégalité sociale ne peut d'ailleurs être altérée sans atteinte à la liberté et à la propriété. L'ordre social, qui repose sur le principe moderne d'égalité assurant les mêmes droits à tous les individus, ne doit alors pas être confondu avec la réalité sociale dans laquelle l'harmonie ne s'an- 
cre pas dans l'égalité mais dans une hiérarchie basée sur la primauté de la propriété. Comme nous le rappelle Fernande Roy dans la conclusion de son ouvrage sur le libéralisme des milieux d'affaires francophones:

Au nom de l'égalité, les droits individuels des travailleurs sont mis sur le même pied que ceux des patrons; l'égalité des droits et des devoirs assure le fondement de l'harmonie qui doit présider à la nécessaire association du capital et du travail. Toutefois, l'affirmation simultanée de la liberté qualifie et limite cette égalité. Juxtaposer ainsi la liberté du capital et celle du travail permet d'évacuer l'antagonisme de ces deux réalités et garantit que la liberté ne détrônera pas la propriété. L'harmonie dont les hommes d'affaires font l'apologie se révèle alors une harmonie entre la tête et les bras, et, quoi qu'ils en disent, cette harmonie s'ancre non pas dans l'égalité mais dans la hiérarchie. À cet égard, elle est bien libérale ${ }^{14}$.

En théorie et en droit, faisant abstraction de la position des individus dans l'échelle sociale, le libéralisme encourage la lutte pour la survie dans les interactions entre des individus postulés comme égaux. Toutefois, afin que l'ordre social soit préservé, le libéralisme préconise davantage la bonne entente et l'harmonie dans les relations entre le capital et le travail, entre les patrons et les ouvriers, entre les riches et les pauvres. En ce sens, tant qu'un individu est ouvrier il ne peut prétendre usurper le rôle du patron et doit accepter sa condition, s'acquitter de son rôle et des devoirs qui en découlent. Toutefois, il est permis à l'individu de tenter d'améliorer sa condition et même d'aspirer à devenir lui-même patron. Il est effectivement du droit de chacun de tenter d'améliorer individuellement sa propre condition. L'individu doit cependant toujours respecter les droits de l'autre ainsi que la propriété privée ${ }^{15}$. 
Confrontée à la pensée religieuse de $\mathrm{M}^{\mathrm{gr}}$ Bruchési, la notion moderne d'égalité ainsi présentée trouve, dans la pratique, un corollaire avec le concept de l'ordre social qui assure l'harmonie entre les hommes par un respect de la place que chacun s'est vu assigner à sa naissance dans les desseins du Créateur. C'est que l'ordre social pour $\mathrm{M}^{\mathrm{gr}}$ Bruchési repose aussi sur une inégalité des conditions où l'harmonie entre les individus et leurs intérêts divergents est assurée par un respect des droits de chacun, selon leur position dans la hiérarchie, ainsi que de la propriété privée. Cependant, à la différence des libéraux, Bruchési ne perçoit pas les inégalités sociales comme le résultat des volontés individuelles dans l'acquisition de la propriété, mais comme le reflet d'une volonté divine afin d'assurer la paix entre les hommes ${ }^{16}$.

L'ordre social, pour l'archevêque de Montréal, possède ainsi une origine transcendante. Fruit de la Création, il est l'incarnation temporelle des desseins de Dieu afin d'assurer la paix entre tous les êtres humains vivant sur la terre. Dieu, dans son absolue sagesse, a constitué le monde de telle façon que tous les hommes puissent vivre dans l'harmonie et dans la paix. Mais, pour que cela se concrétise et que la paix règne sur la terre, les hommes doivent d'abord obéir aux desseins du Créateur en respectant leur rôle et les devoirs qui s'y rattachent. Cette vision du monde qui se dégage des écrits de $\mathrm{M}^{\mathrm{gr}}$ Bruchési nous ramène alors à cet autre fondement de sa pensée thomiste: la hiérarchie sociale.

Sous la diversité apparente du monde, il faut d'abord rechercher l'unité, c'est-à-dire Dieu. Cette unité, constituée par le Créateur, représente le principe structurant de l'univers, du monde, de la société ; un principe qui dépasse l'entendement humain, mais qui est accessible par la révélation divine et auquel l'homme n'a d'autre choix que de se plier. Selon cette logique, tout part de Dieu et tout revient à Dieu. 
Lors de son passage sur la terre, l'homme a une liberté de choix et d'action. Mais sa liberté doit trouver une limite dans les devoirs qu'il doit remplir: des devoirs afin d'assurer le bon fonctionnement de l'ordre instauré par Dieu sur terre, des devoirs en prévision de son retour à Dieu, le Jugement dernier. La fonction et le rôle de chaque être sont donc déterminés par Dieu lui-même qui, lui attribuant une essence, définit la fin de chaque être, son degré de perfection et sa place dans l'univers dont Dieu est la source. L'univers trouve son origine en Dieu et se voit, par conséquent, structuré selon les desseins du Créateur comme une hiérarchie où chaque homme doit occuper la place qui lui a été assignée. Dans cette hiérarchie, le plus bas sert le plus haut tandis que celui-ci guide ou dirige celui-là. Chaque être, en occupant sa place et sa fonction, participe à la perfection de l'univers créé par Dieu. Ainsi, et toujours selon cette logique issue du thomisme, toute société implique, pour fonctionner adéquatement dans l'harmonie et dans la paix, une organisation où règnent des rapports d'autorité : le supérieur doit diriger l'inférieur pour le bien du tout social.

Cette conception hiérarchique des relations humaines qui, dans la pensée religieuse de $\mathrm{M}^{\mathrm{gr}}$ Bruchési possède une origine transcendante, s'accommode pourtant avec la notion moderne d'égalité qui, dans les faits, supporte les inégalités sociales et la hiérarchie qu'entraîne la primauté de la propriété. Tant le libéralisme que le thomisme de l'archevêque s'accordent sur le fait que l'inégalité sociale est chose naturelle, inévitable, que l'être humain se doit d'accepter et de respecter:

Le Christ n'est pas venu détruire l'inégalité des conditions humaines. Cette inégalité est voulue par les desseins de Dieu, son Père. Les lois de la nature en font d'ailleurs une nécessité inévitable. Prétendre bannir de la terre cette inégalité ou s'insurger contre elle, serait 
une chimère. [...] la pauvreté existera toujours, en dépit de toutes les révoltes et de toutes les théories séductrices ${ }^{17}$.

Chacun s'applique alors à défendre la hiérarchie sur laquelle repose l'ordre établi. Les deux camps se confortent ainsi l'un l'autre dans la défense d'un système où l'égalité sociale réelle est rejetée en faveur d'une inégalité naturelle inévitable. Ce faisant, ils deviennent également alliés dans la promotion d'un discours d'harmonie entre la tête et les bras qui vise l'acceptation de la hiérarchie ainsi que l'accomplissement des devoirs liés à la position que l'individu y occupe. Ils se confortent de même mutuellement dans la dénonciation du danger imminent que représente l'idéologie socialiste pour l'ordre social.

En effet, s'accordant au niveau du respect de l'ordre social réel et de ses rapports hiérarchiques, les libéraux et $\mathrm{M}^{\mathrm{gr}}$ Bruchési s'accordent également sur la menace qu'incarne le socialisme pour cet ordre établi. De nombreuses lettres écrites par l'archevêque traitent d'ailleurs de l'erreur fondamentale sur laquelle repose la «funeste utopie »: l'idée de la disparition de l'inégalité sociale et d'une égalité parfaite entre les hommes. Le principal danger de cette idée réside dans l'atteinte à la propriété privée, un des fondements sur lesquels repose la société. C'est que le travailleur, tant qu'il est travailleur, ne peut usurper les pouvoirs du patron. Le socialisme, en voulant déposséder le capitaliste, effectue une usurpation, une spoliation. C'est aller contre le droit à la propriété et contre l'ordre divin. Toutefois, à l'exemple des tenants du libéralisme, $\mathrm{M}^{\mathrm{gr}}$ Bruchési n'est pas contre l'amélioration individuelle des conditions pourvu que cela ne nuise pas à l'ensemble de l'ordre. Ce contre quoi l'archevêque s'élève principalement est la volonté affichée par certains groupes, liés souvent à la classe ouvrière, d'altérer l'ordre social en changeant 
les rapports d'autorité qui y existent. Il est donc impossible, pour une classe sociale tout entière, d'espérer changer son destin sans briser l'ordre et ainsi l'harmonie sociale qui s'y rattache. Les socialistes figurent, dans ce cas-ci, au premier rang des dénonciations dans les écrits de l'archevêque. Cela dit, ce dernier n'est pas contre la revendication des droits légitimes d'une classe sociale par une association comme un syndicat.

À cet effet, l'égalité, au sens libéral du terme, trouve également un corollaire dans les écrits de l'archevêque avec la notion de Justice $^{18}$. Comme nous l'avons déjà mentionné, la notion d'égalité s'exprime par une égalité de droit dans la synthèse libérale. L'État libéral doit assurer cette égalité entre les individus sur lequel repose l'ordre social en droit en garantissant le respect de la justice. Mais dans les faits, l'État doit également veiller à la bonne entente entre des individus ayant une position différente dans l'échelle sociale. Il doit veiller au respect des droits de chacun afin d'éviter toute spoliation ou usurpation. La nécessaire association du capital et du travail repose alors non seulement sur une égalité des droits, mais aussi des devoirs afin d'assurer l'harmonie.

Dans la pensée thomiste de l'archevêque, un équilibre entre les droits et les devoirs est également défendu. De par sa position dans la hiérarchie sociale, l'homme dispose de droits, mais aussi de devoirs qui se rattachent à cette position. Chacun, peu importe sa condition dans la hiérarchie, doit alors vivre selon les droits acquis à cette condition, mais aussi vivre selon les devoirs que lui impose cette dernière. "Les travailleurs, non moins que les capitalistes, assurément ont des droits imprescriptibles. [...] Mais l'ouvrier à l'égal des patrons a aussi des devoirs à remplir. Aux uns comme aux autres, l'Église prêche l'accomplissement de leurs devoirs. Et c'est précisément l'équilibre harmonieux qu'elle cherche à 
établir entre les droits et les devoirs de chacun, qui fait la force de ses enseignements et qui donne à son action une bienfaisante fécondité ${ }^{19}$. " Ainsi, il n'est pas permis à un supérieur hiérarchique d'utiliser les prérogatives propres à sa condition dans le but personnel de s'enrichir ou de nuire à l'autre.

La volonté de Dieu, la loi de l'Évangile, c'est que riches et pauvres, maittres et serviteurs, vivent ensemble dans une harmonie fraternelle, sans colère et sans haine ; mais plein de condescendance les uns pour les autres, respectueux de leurs droits mutuels, unis par les liens de la charité chrétienne, comme les fils d'un même Père, les enfants d'une même famille. En dehors de là, la paix n'est pas possible dans la société $[\ldots]^{20}$.

L'État, ainsi que l'Église, se doit de garantir l'équilibre des droits en assurant le respect de la Justice. D'abord fondée sur le respect de la loi humaine, la notion de Justice, au sein de la pensée religieuse de $\mathrm{M}^{\mathrm{gr}}$ Bruchési, est également une vertu et une loi morale issue de la volonté divine. Elle repose sur un respect de l'ordre social, mais aussi sur le respect de l'autre et des droits propres à sa condition :

La justice est cette vertu qui, par une disposition ferme et durable, incline l'âme à rendre au prochain ce qui lui est dû. Nous vivons en société. Chacun de nous a des droits ; chacun de nous a des devoirs. C'est par l'équilibre de ces droits et l'accomplissement de ces devoirs que l'être social peut se maintenir en des conditions qui lui assurent une existence paisible et un développement régulier ${ }^{21}$.

Désobéir à l'ordre social dans un but strictement personnel, selon $\mathrm{M}^{\mathrm{gr}}$ Bruchési, "c'est réclamer la jouissance de tous ses droits, et commettre la faute grossièrement égoïste de refuser au prochain la liberté de jouir lui aussi des droits qui lui sont propres $^{22} »$. 
En ce sens, il est permis aux individus de se regrouper en associations afin de revendiquer les droits légitimes liés à leur condition. À cet égard, $\mathrm{M}^{\mathrm{gr}}$ Bruchési affirme : "Aucun pouvoir au monde ne saurait vous [les ouvriers] priver légitimement de la faculté de vous former en association particulière. » Il poursuit ensuite en affirmant: "il serait injuste de léser les employés dans le droit naturel qu'ils possèdent, au même titre que tous les citoyens, de se former en association distincte $[\ldots]^{23} »$. Dans le même ordre d'idées, les patrons doivent, pour le bien de l'ordre social, respecter leurs employés et leurs droits: "Si l'on veut que le pauvre se résigne aux misères inséparables de son état, et qu'il soit respectueux des droits d'autrui et de l'ordre social, [...] il faut encore que le capital ne lui refuse aucun de ses droits ${ }^{24}$.»

Reposant a priori sur le respect des droits de chacun ainsi que de la propriété privée, l'ordre social repose aussi sur un devoir de charité chrétienne imposé aux mieux nantis. La charité revêt en effet une importance capitale dans les écrits de l'archevêque. Plusieurs lettres pastorales, mandements et missives personnelles lui sont consacrés. Parente avec la philanthropie libérale, la charité chrétienne vise à assurer un meilleur équilibre des droits, mais aussi à réduire les inégalités sociales afin d'éviter la tentation du socialisme. Elle permet surtout de faire accepter aux classes inférieures leur sort, imposé par la Providence, et ainsi de préserver l'ordre social. Une fois que les droits des ouvriers sont respectés et que les riches remplissent leur devoir de charité, les ouvriers se doivent à leur tour de remplir leur rôle : "une fois que satisfaction est donnée aux justes revendications du travail, l'ouvrier n'a plus de prétexte sérieux à invoquer pour refuser de remplir tous et chacun de ses devoirs à l'égard du patron et de la société. Dans ces conditions de justice et d'apaisement, c'est de la part du travailleur une révolte grave contre les préceptes 
divins et une désobéissance aux lois naturelles que d'entraver le libre exercice des droits du capital ${ }^{25}$." Ainsi, $\mathrm{M}^{\mathrm{gr}}$ Bruchési et les libéraux s'entendent au niveau d'une harmonie entre la tête et les bras et d'un respect mutuel des droits et des devoirs liés à la position qu'occupe l'individu dans une hiérarchie naturelle qui respecte, pour l'un, la volonté divine; pour les autres, la primauté de la propriété.

Pour les libéraux, une autre façon de réduire les inégalités et ainsi de faire accepter la hiérarchie sociale est d'assurer une égalité des chances par l'entremise d'un système d'instruction publique. En effet, l'école publique est un des moyens prônés afin d'égaliser les chances de réussite parmi les enfants provenant de milieux différents. Pour $\mathrm{M}^{\mathrm{gr}}$ Bruchési, l'équilibre dans les droits repose avant tout sur un respect individuel de la vertu de Justice ${ }^{26}$, mais aussi sur l'importance de la charité. L'école publique représente un problème selon l'archevêque, d'une part, en raison des conflits de juridiction qu'elle risque de déclencher avec l'État, d'autre part, à cause du faux sentiment d'égalité qu'elle pourrait diffuser au sein de la population. Ce sentiment est dangereux puisqu'il peut conduire à l'envie et à la jalousie. Ajouté au sentiment de liberté exagérée, il conduit la société vers un état constant de rivalité où tous les individus doivent lutter les uns contre les autres afin de s'assurer une meilleure place dans la hiérarchie. Si chacun accepte le sort que lui réserve la Providence, il n'y aura alors pas d'envie et tous les individus pourront vivre dans la paix et dans le respect mutuel des droits de chacun.

Contrairement à ce qui est véhiculé par la modernité27, la lutte entre les individus et leurs divergences d'intérêts ne sont pas une chose naturelle que l'État doit réguler. Pour $\mathrm{M}^{\mathrm{gr}}$ Bruchési, les êtres humains doivent vivre selon la condition qu'ils se sont vu offrir dans la hiérarchie par leur naissance et contrôler leurs passions. La hiérarchie sociale est d'ailleurs le 
reflet de l'intervention divine afin que tous les hommes puissent vivre dans la paix. Alors, malgré les accommodements et les ententes pratiques, une tension demeure au niveau théorique entre la notion moderne d'égalité et la hiérarchie sociale thomiste. Cette tension repose sur une conception séculière de l'égalité qui, tout comme la notion moderne de liberté, est fondée sur l'évacuation du caractère transcendant de l'existence humaine ainsi que de la révélation. Dans la pensée thomiste de l'archevêque, l'égalité entre les hommes n'est pas fondée sur une égalité de nature dans les chances de s'enrichir, mais bien sur une égalité dans les possibilités d'accéder au Ciel et d'obtenir le salut. Tous les hommes sont donc égaux devant Dieu, mais son socialement inégaux de par leur nature, leur essence. Cette nature étant voulue par Dieu luimême, les inégalités sociales existant au sein de la société sont le reflet temporel d'un dessein divin qui dépasse les simples volontés individuelles.

Plus spécifiquement, cette tension au niveau théorique entre l'égalité moderne et la hiérarchie sociale thomiste nous ramène à cette différence fondamentale entre le libéralisme et le catholicisme au début du siècle : une différence entre une conception atomisée et sécularisée de la société où l'homme est le centre de toute chose autour duquel se construisent les structures sociétales et une conception organique dans laquelle l'homme doit trouver la place qui lui revient dans un univers structuré hiérarchiquement à partir d'un principe transcendant. Faisant de l'individu libre et égal aux autres le fondement ontologique de la société, le libéralisme renvoie l'organisation sociale et politique à la responsabilité individuelle d'une multitude d'individus qui, en lutte les uns contre les autres dans l'acquisition de la richesse, travaillent néanmoins ensemble afin de bâtir une société et des lois qui protégeront l'individu et ses droits naturels. Pour le catholicisme 
qui place Dieu comme fondement de la société, l'individu ne constitue pas la norme de toute chose et l'organisation politique et sociale se voit attribuer une origine transcendante qui surpasse l'entendement humain et à laquelle l'individu doit se plier pour le bien du plus grand nombre. Ce faisant, en théorie, la société organique de $\mathrm{M}^{\mathrm{gr}}$ Bruchési, reposant sur une vision paternaliste des relations humaines où l'individu doit se conformer au sort de la Providence et obéir aux exigences qui en découlent, rejette une vision sociale qui repose principalement sur une interaction sécularisée entre des individus libres et égaux de nature, en compétition pour la réussite personnelle. Il affirme à cet égard :

Plus le sens de la justice s'éveille dans la conscience publique, plus les faiseurs les moins scrupuleux s'abstiendront de rien entreprendre qui porte atteinte au caractère de probité et d'honorabilité des relations et des transactions. Le serment sera pour tous chose religieuse et sacrée. On subordonnera l'intérêt propre à l'intérêt commun. On fera de l'intégrité des droits la base inviolable de l'édifice social. Et les droits de chacun, reconnus et inviolés, engendreront le respect mutuel, la confiance réciproque, la rectitude des procédés et la dignité des attitudes ${ }^{28}$.

\section{La propriété}

Dans la synthèse libérale, la notion fondamentale de l'égalité, bien que très importante, demeure en définitive une valeur subordonnée à la propriété. En fait, exprimées ensemble, les notions fondamentales de liberté, d'égalité et de propriété se retrouvent néanmoins ordonnées dans une hiérarchie qui leur donne leur signification et leur limite. Comme le mentionne d'ailleurs Fernande Roy: 
Si le principe de l'égalité est nécessaire pour affirmer celui de la liberté, l'égalité des individus dans le droit à la liberté, il semble bien qu'à son tour le principe de la liberté vienne étayer celui de la propriété : la liberté permet à l'individu d'assurer son bonheur dans l'épanouissement de sa propriété [...] La revendication de la liberté est essentielle parce qu'il faut être libre pour être propriétaire ; mais, parce qu'on n'est pas pleinement libre sans être propriétaire, c'est la propriété qui représente la fin, et la liberté, le moyen ${ }^{29}$.

Nous n'avons donc qu'à rappeler l'importance centrale de la propriété dans le libéralisme. Elle est en définitive l'élément structurant sur lequel repose l'ordre libéral. Tout comme la notion de liberté, la propriété se présente comme un droit fondamental issu de la volonté de l'homme de posséder des biens. Liée directement à la notion de liberté, la propriété apparaît surtout, au sein du libéralisme, comme l'extension ou la matérialisation de la liberté individuelle et de la volonté personnelle. Perçue comme le principal moyen d'assurer le bonheur, la propriété se présente également comme le moteur essentiel à toute activité humaine : l'individu poursuivant son intérêt personnel dans l'acquisition de la propriété sert la société dans son ensemble en accroissant la richesse collective. Au sein des sociétés libérales et industrielles, la notion de propriété est intimement liée à une valorisation du travail. Par son travail, son savoir-faire, sa science, l'homme transforme la nature et lui donne une utilité en la transformant en biens. Par ce même travail, l'homme peut acquérir des biens et ainsi de la propriété.

Confrontée à la pensée de $\mathrm{M}^{\mathrm{gr}}$ Bruchési, la notion de propriété ainsi présentée constitue à la fois un point d'entente et une grande source de discorde. Entente d'une part, en raison de l'importance accordée à la protection de la propriété privée dans la pensée de l'archevêque, discorde d'autre 
part, en raison de la fixation matérialiste qui peut en découler, fixation égoïste à la source même du « Mal». Débutons avec le point d'entente.

Selon la conception thomiste de $\mathrm{M}^{\mathrm{gr}}$ Bruchési, le droit à la propriété privée constitue un droit fondamental, un droit accordé à l'homme par Dieu. Depuis l'encyclique Rerum novarum de Léon XIII, la doctrine catholique s'est efforcée d'offrir une interprétation de la tradition par rapport à la propriété qui tente de répondre aux réalités du libéralisme économique et du capitalisme en développement. Il s'agit principalement d'une stratégie doctrinale qui vise à "associer» les conceptions thomistes de la propriété aux idées et aux aspirations libérales. La propriété est donc reconnue comme essentielle à l'être humain qui doit utiliser les choses de ce monde mises à sa disposition pour vivre. Dieu a créé ces choses afin que l'homme puisse les dominer et les posséder. C'est la destinée de l'homme de s'en emparer et de les utiliser afin d'assurer son bien-être lors de son passage sur la terre. $\mathrm{M}^{\mathrm{gr}}$ Bruchési donne également une grande importance au travail qui assure à l'homme la juste possession de la propriété. Il est donc interdit à qui que ce soit de s'en prendre à la propriété privée acquise selon les justes conditions du travail, que ce soit par une personne ou par une instance élevée.

En ce qui a trait maintenant au point de discorde, celui-ci trouve principalement ses origines dans ce que $\mathrm{M}^{\mathrm{gr}}$ Bruchési perçoit comme une fixation matérialiste liée à la recherche obsessive des choses de ce monde. D'abord, avant la propriété privée, $\mathrm{M}^{\mathrm{gr}}$ Bruchési défend l'idée d'une conception commune des biens terrestres donnés par Dieu pour la jouissance de tous. La Justice et la Charité exigent que toutes richesses superflues soient utilisées pour subvenir aux besoins des pauvres. Il ne s'agit pas d'un refus de la conception privée de la propriété, mais plutôt une dénonciation de la concentra- 
tion abusive de la richesse entre les mains de la classe dirigeante qui, par vanité et par avarice, refuse injustement ou oublie simplement de répondre à ses devoirs de charité chrétienne. Ensuite, plus que dans le plaisir d'avoir ou d'acquérir des biens, le véritable bonheur de l'homme doit se trouver dans le salut. $\mathrm{M}^{\mathrm{gr}}$ Bruchési s'élève donc contre une conception de la vie qui vise à faire de la propriété la finalité à atteindre, l'objectif même de l'existence, le moteur de toutes activités. Selon ce dernier, l'existence doit reposer sur des objectifs beaucoup plus nobles tels l'aspiration à faire le Bien, le devoir de mener une vie juste et honorable selon les Saints Enseignements et, surtout, le désir de retourner vers le Créateur. Par conséquent, le travail ne doit pas viser exclusivement le gain, mais doit répondre aussi à la nécessité de remplir ses justes devoirs envers la société. Pour l'archevêque, le travail doit d'abord être perçu comme un devoir lié à la condition de l'ouvrier, mais aussi comme un moyen de pénitence afin d'expier ses fautes. Il est intéressant de souligner ici un lien fait par $\mathrm{M}^{\mathrm{gr}}$ Bruchési dans la lettre pastorale Sur la question ouvrière entre le travail, les ouvriers et la souffrance du Christ. Le travail est alors considéré, à l'instar de l'expérience de Jésus, comme une souffrance nécessaire au bien du plus grand nombre.

Les réserves à l'égard de la notion fondamentale de propriété dans la pensée religieuse de l'archevêque viennent non pas d'un refus formel de toute richesse ou de toute possession, mais bien de la condamnation de l'abus des choses de ce monde. Il récuse une conception strictement matérielle de l'existence qui détourne les yeux de l'homme du ciel et qui le sépare du sens véritable de la vie, le sens spirituel. À cet effet, les écrits de l'archevêque regorgent, débordent même de mises en garde contre la passion de la convoitise et le désir abusif d'accumulation qui poussent les individus à se laisser aller 
dans l'assouvissement coupable de tous les désirs. Il s'agit bien là d'un problème majeur pour $\mathrm{M}^{\mathrm{gr}}$ Bruchési ; à tel point qu'il semble faire de celui-ci la source principale de tous les malheurs qui menacent la société moderne à l'aube $\mathrm{du} \mathrm{XX}^{\mathrm{e}}$ siècle.

De fait, la source du «Mal» chez $\mathrm{M}^{\mathrm{gr}}$ Bruchési parait résider dans une abondance matérielle démesurée appuyée par une philosophie utilitariste qui tend à faire du monde une source de biens mis à la disposition de l'homme et dans laquelle ce dernier peut puiser à volonté. Le désir de la possession et la passion de l'acquisition sont dénoncés comme la source même de toutes les «erreurs modernes » qui poussent les individus à l'assouvissement de toutes les passions et à l'oubli des saines traditions et des devoirs chrétiens.

Selon l'archevêque, il faut "trouver la ligne de démarcation entre l'usage et l'abus gravement périlleux des choses de ce monde ${ }^{30} »$. Il s'agit de trouver une limite entre l'utilisation convenable des biens mis à la disposition de l'homme par la société moderne et l'excès qui tend à faire de ce dernier un animal, une machine vouée à la recherche insatiable de tous les plaisirs du corps. Surtout, il s'agit de faire de la propriété un moyen et non une fin et ainsi éviter que l'homme ne devienne un individu défini essentiellement par ce qu'il possède et motivé principalement par ce qu'il désire posséder. La notion fondamentale de propriété revêt donc une importance capitale dans la compréhension de la perception bruchésienne de la modernité. Utilisée convenablement, la propriété est perçue comme étant essentiellement positive puisqu'elle permet à l'homme d'améliorer son existence terrestre. Convoitée excessivement, elle est la source principale des grands malheurs modernes puisqu'elle détourne l'homme de sa véritable nature et le pousse sur les chemins de la facilité et de l'individualisme passionnel, égoïste et narcissique. 


\section{La psychologie de l'individualisme}

L'un des traits fondamentaux de la modernité est l'individualisme ou, pour reprendre les termes employés par le théologien William Edgar: "la conscience moderne de la valeur que revêt la personne individuelle face à des entités plus larges susceptible de l'amputée ${ }^{31}$ ". Cet individualisme, ainsi que le libéralisme, son prolongement logique, visent l'épanouissement de l'être humain par la liberté individuelle et l'exercice d'une responsabilité personnelle ${ }^{32}$. Ce principe psychologique est lui-même lié à la notion de subjectivité qui, dans la modernité, tend à faire de l'individu un être raisonnable, apte à prendre des décisions pour lui-même sans l'intervention d'une entité plus grande ${ }^{33}$. Comme nous l'avons mentionné dans la section portant sur la notion de liberté, $\mathrm{M}^{\mathrm{gr}}$ Bruchési alloue une certaine subjectivité à l'être humain dans la mesure où l'encadrement des fidèles consiste à suggérer la limite à ne pas dépasser dans le libre exercice de la responsabilité individuelle. Cette limite doit cependant tenir compte, selon la conception thomiste de l'archevêque, de la dimension spirituelle de la personne humaine. Ainsi présentée, la notion de subjectivité semble alors trouver un corollaire dans le rôle primordial que joue la conscience chez l'individu.

Pour $\mathrm{M}^{\mathrm{gr}}$ Bruchési, tous les individus sont dotés d'une conscience : une faculté qu'a l'homme de connaître sa propre réalité et de la juger lui-même. Intrinsèquement liée à la raison, elle permet à l'homme d'effectuer lui-même ses propres choix concernant sa propre existence en ayant recours à un jugement personnel. Plus largement, la conscience apparait, chez $\mathrm{M}^{\mathrm{gr}}$ Bruchési, comme une sorte d'autorité morale intérieure présente en chaque individu qui lui permet d'exercer son jugement et le guide. Elle s'affiche alors, d'une part, comme une faculté d'autodétermination qui assure l'exercice d'un jugement personnel et d'une liberté de choix et, d'autre 
part, comme une faculté morale qui doit aussi assurer une responsabilité personnelle devant le salut éternel. D'où l'existence de l'enfer et du purgatoire dans la mythologie chrétienne qui incarne la part punitive dans l'éventualité d'une existence parsemée de choix mauvais et d'erreurs de conscience. Avec la raison, l'importance de la conscience personnelle dans la pensée thomiste de $\mathrm{M}^{\mathrm{gr}}$ Bruchési rend possible l'accommodement avec la psychologie de l'individualisme dans la mesure où celle-ci n'est pas séparée du principe transcendant duquel elle tire son essence ${ }^{34}$. À cet égard, il n'est pas surprenant que la principale source de tension au niveau de la conception moderne de l'individualisme chez l'archevêque de Montréal se trouve dans l'affirmation d'une conscience individuelle qui s'édifie sur la négation de la révélation et de l'inévitabilité du Jugement dernier. Il s'agit de la subjectivité départie de l'élément transcendant et de la moralité qui en découle : une subjectivité sécularisée.

En effet, dans la modernité, la psychologie de l'individualisme trouve sa justification dans une conception de l'univers qui tend à consacrer l'individu comme étant la norme de toute chose à partir de laquelle le monde s'est ensuite constitué : l'individu libre représente alors le fondement ontologique de toute société. De cette perception moderne de l'individu se dégage aussi une orientation, une disposition vers un anthropocentrisme où, pour s'affirmer pleinement libre, l'homme doit se libérer du poids du ciel et des traditions qui s'y rattachent afin de se concentrer principalement sur son existence terrestre et matérielle, avant tout garante de la réalisation personnelle. Cette orientation anthropocentrique agissant au sein de l'individualisme moderne s'affirme au détriment de la conception théocentrique sur laquelle repose la pensée thomiste de $\mathrm{M}^{\mathrm{gr}}$ Bruchési et selon laquelle l'homme, occupant une place centrale dans le monde d'ici-bas qu'il con- 
tribue à construire, est lui-même ordonné à une réalité plus haute dont Dieu est le centre. D'où le devoir absolu de s'inspirer de la Révélation divine et de chercher le salut de son âme. L'homme ne peut donc se défaire volontairement des nécessités que lui imposent les traditions, celles-ci étant porteuses des Enseignements divins communiqués par un Dieu tout-puissant qui se manifeste dans la Révélation et auxquels on se doit d'être attentif, par le biais de la conscience, pour le maintien de l'harmonie sur terre. Dieu est donc là, présent dans la vie de tous les jours. Il observe. Il communique avec l'homme. Ses enseignements ne peuvent être méprisés qu'au prix de grandes souffrances et de catastrophes sociales.

La tendance anthropocentrique qui s'affirme au sein de l'individualisme moderne constitue le Mal à abattre pour l'archevêque de Montréal. La source d'une pareille conscience individualiste décentrée qui tend à se détourner des Enseignements divins semble se trouver dans une abondance matérielle qui détourne les yeux de l'homme du ciel afin de les fixer uniquement sur les biens matériels convoités. Ainsi, $\mathrm{M}^{\mathrm{gr}}$ Bruchési se dresse devant l'émergence d'une conception libérale de l'homme de plus en plus en vogue au début du siècle et qui tend à faire essentiellement de l'individu et de sa propriété la fin suprême de toute société.

À cet effet, la lettre pastorale sur L'affaiblissement de l'esprit chrétien et le goût des plaisirs du monde (1901) est particulièrement utile pour saisir la vision bruchésienne de l'individualisme moderne. Bien que l'intention première de cette lettre réside dans la volonté de prévenir les fidèles du diocèse de Montréal contre les dangers du théâtre étranger, $M^{{ }^{g x}}$ Bruchési dépasse la simple dénonciation en se prononçant clairement, et ce dès le début, contre ce qu'il perçoit comme étant la source de tous les malheurs qui menacent la famille ainsi que la société canadienne-française à l'aube du $\mathrm{XX}^{\mathrm{e}}$ siècle. L'archevê- 
que poursuit ensuite, sur plusieurs pages, en développant de façon détaillée et élaborée ce qui semble être un véritable schéma analytique de causalité visant à expliquer le cheminement de la désintégration des valeurs morales et traditionnelles, à partir de cette source, jusqu'à l'effondrement de la communauté familiale constituée selon les desseins de Dieu.

Monseigneur Bruchési se contente d'abord de décrire la source de ce Mal comme l'« atmosphère de mollesse et de relâchement, l'attrait des frivolités du siècle, la fièvre des plai$\operatorname{sirs}^{35} »$. Ce que l'archevêque constate surtout c'est que les "mœurs modernes» ne sont pas porteuses de cette limite à l'exercice de la liberté individuelle construite autour des idéaux de devoir, de tempérance et de sacrifice qui constituent, pour ce dernier, les valeurs fondamentales d'une société. L'homme, incité sans arrêt au divertissement dans une société de consommation et encouragé par un matérialisme qui flatte son appétit sensitif, s'engage dans une fausse quête d'un paradis sur terre où l'accumulation de biens et de jouissances devient la visée première de tout être:

Se soustraire, d'une part, avec une sorte d'horreur instinctive, aux moindres privations, aux plus légers sacrifices, à tout effort pénible; d'autre part, s'ingénier à ne perdre aucune occasion de goûter aux joies de la vie, s'exaspérer même et se révolter de ne pouvoir briller ou jouir autant que les autres; et, pour satisfaire ces aspirations, s'épuiser dans une recherche des biens de la terre qui absorbe les principales facultés de l'intelligence et du cœur ; n'est-ce pas là se faire une idée fausse des conditions de l'existence ici-bas ${ }^{36}$ ?

S'attaquant à l'homme par la stimulation de ses instincts et de ses passions, les "mœurs modernes » encouragent l'égoisme propre à la nature humaine, assurent la domination des passions sur la raison et poussent alors l'homme à se dérober 
aux devoirs que le Créateur lui a imposés. Le premier devoir à souffrir de cette quête du bonheur matériel n'est nul autre que le rôle primordial de parent :

La mère n'est plus cette vraie femme chrétienne, cette femme forte dont parle l'Écriture. Elle ne vit plus uniquement pour son époux et pour ses enfants. Sa vie se répand au dehors, emportée par des inclinations profanes, réclamée par des sollicitudes mondaines. Son cœur, partagé entre ses devoirs d'état si beaux et si sanctifiants, et les exigences toujours absorbantes et parfois si dangereuses de la société moderne, ne sait plus fournir cette surabondante provision de tendresse et d'abnégation, absolument nécessaire au maintien d'une union affectueuse parmi les membres de la même famille.

$M^{\mathrm{gr}}$ Bruchési poursuit ensuite en affirmant :

Le père, de son côté, n'est pas toujours le chef auguste et fort de la société domestique. Pressé qu'il est de réaliser ses rêves de grandeurs et d'opulence, mal venu qu'il serait de refuser à son épouse et à ses enfants des vanités et des divertissements dont il se montre luimême trop avide ; l'énergie de sa volonté comme de son esprit s'étiole dans une activité fébrile et une existence mal réglée. Ce n'est plus un sage modérateur, un gardien fidèle et dévoué, un maître aimé et respecté. Il est fatalement condamné à déchoir du noble rôle que la Providence lui avait assigné ${ }^{37}$.

Porté par ses passions et dirigé par ses vanités et ses appétits de grandeur dans une vie de liberté exagérée, l'homme, selon $\mathrm{M}^{\mathrm{gr}}$ Bruchési, n'écoute plus la voix de sa conscience et en arrive même à perdre le sens du devoir : devoir qu'il doit remplir envers lui-même et la société. Surtout, dans la lettre pastorale sur L'affaiblissement de l'esprit chrétien et le goût des plaisirs $d u$ monde, l'archevêque insiste sur la disparition du devoir 
parental et, avec lui, sur la désintégration de la famille. «Dans ces conditions, l'intimité conjugale doit disparaître. Elle s'en va, en effet, entraînant après elle ses saintes solidarités, ses doux épanchements et ses invincibles préservatifs. C'est le signal de la désagrégation et de l'indépendance réciproque ${ }^{38}$. » Dépourvu de l'enseignement privilégié des valeurs chrétiennes qu'il aurait reçu au sein d'une famille unie par les liens de l'amour, du respect et de l'obéissance, l'enfant, laissé à luimême et privé de modèle parental fort et vertueux, ne peut que se laisser entraîner sur la voie dangereuse d'une vie dirigée par l'assouvissement sans restriction de tous les désirs du corps. «Cette dégradation, affirme Bruchési, ce règne des sens [...] c'est l'asservissement de l'esprit au corps, la perte de la pudeur, la tyrannie des passions ; l'affaiblissement du caractère, le dégoût du devoir, de la piété et de la vertu ${ }^{39}$.»

\section{$* * *$}

L'analyse des différentes notions libérales fondamentales présentes dans les écrits de $\mathrm{M}^{\mathrm{gr}}$ Bruchési nous a permis de constater que le « $\mathrm{Mal}$ » repose sur une vision erronée de la vie visant à détacher l'homme de son Créateur et à faire de celui-ci un animal dominé par ses désirs et ses instincts ${ }^{40}$. C'est contre une "mauvaise voie », marquée par un individualisme possessif libéré en tant que tel du salut éternel que s'élève $\mathrm{M}^{\mathrm{gr}}$ Bruchési. Ce que l'archevêque craint par-dessus tout est l'émergence d'une société atomisée, composée d'hommes détachés de l'autorité des traditions, centrés sur eux-mêmes, délaissant devoirs et honneur et n'ayant comme préoccupation que la réussite matérielle. L'individualisme anthropocentrique et matérialiste, parce qu'il présente une trop grande liberté en niant l'aspect spirituel de l'existence humaine et les Saints Enseignements et parce qu'il consacre la domination du corps sur la raison, est donc la notion, la tendance mo- 
derne qui déclenche les plus grandes appréhensions chez $\mathrm{M}^{\mathrm{gr}}$ Bruchési. Dans une plus large mesure, il est possible d'affirmer que cette notion, liée au libéralisme philosophique, définit la nature même du rapport entre l'orthodoxie catholique et la modernité au début du siècle puisque c'est principalement à partir de cette notion que $\mathrm{M}^{\mathrm{gr}}$ Bruchési perçoit et dénonce la modernité en ce qu'elle a de répréhensible à ses yeux.

Il s'agit de cette "mauvaise voie », la voie de la facilité plutôt que celle du devoir, la voie du corps plutôt que celle de la raison, qui menace l'homme et la société moderne. Pour $\mathrm{M}^{\mathrm{gr}}$ Bruchési, cette tendance qui s'exprime au sein des idéaux modernes représente la voie de la tentation offerte par Satan afin de séduire les faibles. Ceux dont l'esprit, la foi ou la raison est faible se laissent tenter par une vie consacrée au plaisir du corps et à l'assouvissement de tous les instincts. Alors, ce que $\mathrm{M}^{\mathrm{gr}}$ Bruchési perçoit dans la diffusion de l'individualisme moderne est la transmission du " Mal», transmission qui se traduit ensuite par un abandon progressif des valeurs et des vertus qui font de l'homme un être fort, un être spirituel $^{41}$. Une fois séduit par la voie de la facilité, par la tentation de Satan, l'homme ne peut revenir en arrière : il devient un animal, un être corrompu ayant perdu le sens des vertus honorables et qui ne pense qu'à assouvir ses désirs plutôt qu'à accomplir ses devoirs. Il devient un homme sournois, menteur et manipulateur prêt à n'importe quoi pour satisfaire sa soif de plaisir et ses ambitions de réussite personnelle. Pire encore, une fois séduit et lancé sur cette voie, le " corrompu » ou le «malhonnête » tente à son tour de séduire ceux qui n'ont pas encore quitté le chemin de la raison et de la vertu. Persuadé que la voie du corps est de loin meilleure parce que plus facile et plus naturelle, les "corrompus » ou les «méchants ", dénoncés par $\mathrm{M}^{\mathrm{gr}}$ Bruchési, s'appliquent à entrainer les raisonnables avec eux, sur les chemins qui mènent vers l'enfer ${ }^{42}$. 
Ceci dit, l'accommodement est possible avec un libéralisme qui, dans la réalité historique du début du $\mathrm{XX}^{\mathrm{e}}$ siècle, n'émerge pas totalement à contresens de la tradition, mais qui s'appuie plutôt sur cette dernière pour se réaliser. Une entente pratique et tacite est réalisable avec un libéralisme historique qui nécessite un élément d'ordre afin de protéger l'individu et sa propriété ; un libéralisme pratique qui se distingue du libéralisme philosophique par la survivance et la tolérance en son sein de certains éléments de la tradition. L'hostilité et la crainte manifestées par $\mathrm{M}^{\mathrm{gr}}$ Bruchési dans ses écrits sont donc dirigées moins contre le libéralisme tel qu'il s'est réalisé dans la réalité historique, que contre ce vers quoi il tend, c'est-à-dire l'horizontalité de toute la vie et la négation de la révélation et du salut éternel ainsi que des devoirs qui en découlent. Les foudres de l'archevêque sont alors dirigées principalement contre un libéralisme philosophique et théorique qui risque de provoquer la désintégration d'un système de valeurs traditionnelles reposant essentiellement sur les notions d'honneur et d'obéissance au profit d'une "voie erronée » basée sur la promotion d'une "mauvaise liberté ». Elles sont dirigées contre un individualisme égoïste et matérialiste qui tend à l'abandon des vertus qui, au sens où $\mathrm{M}^{\mathrm{gt}}$ Bruchési l'entend, distinguent l'homme de l'animal, consacrent sa nature spirituelle et assurent le bon fonctionnement social.

\section{NOTES}

${ }^{1}$ André Vachon, «Chronologie de $\mathrm{M}^{\mathrm{gr}}$ Paul-Napoléon Bruchési », Revue de l'Université Laval, 20 (décembre 1965), p. 352-368.

${ }^{2}$ Jean Bruchési, «L'abbé Paul-Napoléon Bruchési à Québec (1880-1884)», Cabiers des Dix, vol. 21 (1956), p. 138-139.

${ }^{3}$ Vachon, «Chronologie de M"r Paul-Napoléon Bruchési », p. 353-355. 
4 Jean Bruchési, «Un inlassable épistolier : Paul-Napoléon Bruchési (18551939) ", Mémoires de la Société Royale du Canada, vol. 10 (1972), p. 116.

${ }^{5}$ Nous faisons référence ici aux nombreuses études sur la transformation des mœurs au Québec qui traitent de la lutte contre l'immoralité de $\mathrm{M}^{\mathrm{gr}}$ Bruchési : Pierre Hébert, Censure et littérature au Québec, Montréal, Fides, 1997, 252 p. ; Yves Lever, L'histoire générale du cinéma au Québec, Montréal, Boréal, 1995, 635 p. ; Mireille Barrière, «Le goupillon, le maillet et la censure du théâtre lyrique à Montréal (1840-1914) ", Les Cabiers des Dix, vol. 54 (2000), p. 119-135 ; Michel Bellefleur, L'Église et le loisir au Québec avant la Révolution tranquille, Montréal, Presses de l'Université du Québec, 1990, 221 p. ; Jean Laflamme et Rémi Tourangeau, L'Église et le théâtre au Québec, Montréal, Fides, 1979, 355 p. ; Lise Saint-Jacques, $M^{\text {rr }}$ Bruchési et le contrôle des paroles divergentes : journalisme, polémique et censure (1896-1910), Mémoire de M.A. (histoire), UQAM, 1987, 140 p.

${ }^{6}$ C'est l'interprétation qui se dégage de la synthèse historique, Linteau, Durocher et Robert, Histoire du Québec contemporain. De la Confédération à la crise (18671929). Montréal, Boréal, 1989. Le chapitre portant sur l'Église et l'école, en particulier, traite des résistances de $\mathrm{M}^{\mathrm{gt}}$ Bruchési à la bibliothèque publique, à l'éducation obligatoire et au ministère de l'Instruction publique. C'est aussi ce qui se dégage des travaux de l'historien Yvan Lamonde qui, à travers ses nombreuses publications, tenta de démontrer la continuité d'une tradition libérale radicale au Québec après la disparition des rouges. Dans un ouvrage collectif dirigé par Lamonde et intitulé Combats libéraux au tournant $d u X X^{\circ}$ siècle (Montréal, Fides, 1995), les auteurs s'appliquent à démontrer comment les tenants du libéralisme radical ont dû combattre les éléments traditionnels de la société québécoise, notamment l'archevêque de Montréal, afin d'en permettre la modernisation.

${ }^{7}$ Plusieurs théoriciens ou philosophes tels Hobbes, Spinoza ou Locke ont contribué, par leurs réflexions sur les institutions humaines, à façonner le fondement de la conception de l'homme moderne qui réside en la liberté de l'individu de faire ce qu'il désire. Dans son ouvrage intitulé Leviathan (1651), Thomas Hobbes affirme que l'homme est une machine naturelle dotée de deux principales qualités : le désir et l'agir. À l'état naturel, l'homme est libre de poursuivre l'objet de ses passions et d'acquérir tout de ce qui est nécessaire afin de préserver sa vie. S’appuyant sur Descartes et sur l'école du Droit naturel, Hobbes fait également siennes les idées selon lesquelles la vérité sur le monde ne serait pas seulement l'apanage de la tradition ou des autorités religieuses car chaque individu peut, grâce à sa raison, découvrir la vérité. 
${ }^{8}$ " Lettre pastorale de $\mathrm{M}{ }^{\mathrm{r}}$ Paul Bruchési sur l'affaiblissement de l'esprit chrétien et le goût des plaisirs du monde ", 16 décembre 1901, Mandements, tome 13, Montréal, Arbour et Dupont, p. 445-446.

${ }^{9}$ Le concept de dignité humaine est un concept qui revient constamment dans la correspondance de l'archevêque. La dignité de l'homme est directement liée à son caractère spirituel : il est la seule créature terrestre dotée d'une âme spirituelle et immortelle. De cette dignité découle la liberté de l'homme, une liberté de nature certes, mais qui doit tenir compte du rapport de l'homme avec son Créateur. Toute nature, même non spirituelle, est une création de Dieu et reste soumise au Législateur suprême.

${ }^{10}$ Selon $\mathrm{M}^{g t}$ Bruchési, la juste liberté est celle qui ne sépare pas l'homme des devoirs chrétiens qu'il doit remplir et des saines traditions qu'il doit respecter.

${ }^{11}$ Humilité, force, tempérance et honneur sont des valeurs qui reviennent constamment dans la correspondance de l'archevêque. Elles sont tous liées à un concept de "contrôle " qui vise à faire de l'homme un être sain par la maitrise des passions et des envies issues du corps. Elles sont directement liées à la notion de devoir que l'homme se doit de respecter : devoir de l'homme envers lui-même, envers la société et envers Dieu.

${ }^{12}$ «Lettre pastorale des Pères du premier Concile Plénier de Québec : L’esprit chrétien dans l'individu, dans la famille et dans la société ", $1^{\text {cr }}$ novembre 1909, Mandements, tome 14, Montréal, Arbour et Dupont, p. 564.

${ }^{1.3} \mathrm{M}^{\mathrm{gr}}$ Paul Bruchési, « sur l'affaiblissement de l'esprit chrétien... », tome 13, p. 446.

${ }^{14}$ Fernande Roy, Progrès, harmonie, liberté. Le libéralisme des milieux d'affaires francophones à Montréal au tournant du siècle. Montréal, Boréal, 1988, p. 272.

${ }^{15}$ Fernande Roy nous rappelle avec justesse que, dans la synthèse libérale, l'égalité demeure une valeur subordonnée, soumise à la propriété : « [ . . . l'égalité libérale supportera en particulier les inégalités dans la réussite de l'exercice de la liberté. La liberté individuelle, en effet, ne permet pas qu'une autorité extérieure vienne, au nom d'un principe collectif, imposer une égalité sociale ». Roy, ibid., p. 50.

${ }^{16}$ En ce qui a trait au concept de l'ordre social dans la pensée de $\mathrm{M}^{\mathrm{gr}}$ Bruchési, il convient de souligner l'importance de la lettre pastorale Sur la question ouvrière où l'archevêque demande aux ouvriers et aux patrons de la ville de Montréal de respecter l'ordre social voulu par Dieu ainsi que les droits et les devoirs qui 
se rattachent à leur place respective dans celui-ci. Plusieurs citations utilisées dans cette section sur l'égalité sont d'ailleurs tirées de cette lettre pastorale.

${ }_{17}$ "Lettre pastorale de $\mathrm{M}^{\mathrm{gt}}$ Paul Bruchési sur la question ouvrière ", 23 avril 1903, Mandements, tome 13, Montréal, Arbour et Dupont, p. 527.

${ }^{18}$ Dans la lettre Sur la Justice (4 novembre 1915), M ${ }^{\mathrm{gr}}$ Bruchési explique la nécessité sociale de l'équilibre des droits. Dans cette lettre pastorale, $\mathrm{M}^{\mathrm{gr}}$ Bruchési traite de l'importance du respect des droits et des devoirs de chacun afin d'assurer le bon fonctionnement social des individus. « Lettre pastorale de $\mathrm{M}^{\mathrm{gr}}$ Paul Bruchési sur la justice », Mandements, Tome 15, Montréal, Arbour et Dupont, p. 351-366.

${ }^{19} \mathrm{M}^{\mathrm{gr}}$ Paul Bruchési, «Sur la question ouvrière », tome 13, p. 518.

2) Ibid., p. 527.

${ }^{21} \mathrm{M}^{\mathrm{gr}}$ Paul Bruchési, « Sur la justice », tome 15, p. 355.

${ }^{22} \mathrm{M}^{\mathrm{gr}}$ Paul Bruchési, «Sur la question ouvrière », tome 13, p. 531.

${ }^{23}$ Ibid., p. 530-531.

${ }^{24}$ Ibid., p. 530.

${ }^{25}$ Ibid., p. 531.

${ }^{26} \mathrm{Si}$ un individu sent que les droits liés à sa condition ne sont pas respectés, il peut alors faire le nécessaire afin de revendiquer ce qui lui revient. Évidemment, la revendication doit toujours se faire pacifiquement, de prime abord par la discussion. Il est d'ailleurs interdit, au nom de la Justice, à un supérieur de refuser les droits d'un inférieur. $\mathrm{M}^{\mathrm{gr}}$ Bruchési affirme que l'État et l'Église se sont toujours faits les défenseurs des gagne-petit afin d'éviter toute injustice à leur égard. Mais une fois que satisfaction est donnée aux justes revendications de l'inférieur, celui-ci n'a plus de prétexte pour refuser de remplir ses devoirs à l'égard de la société.

${ }^{27}$ Selon le mythe fondateur de la modernité, il y aurait, avant la morale, la loi et l'État, un état naturel où les hommes auraient été libres, égaux et rationnels. Dans ce mythique état de nature il n'existe donc que des individus qui ne peuvent être compris à partir d'une totalité, fût-elle la société ou le Créateur. À l'inverse, la totalité doit être comprise à partir de l'individu libre et égal qui devient le fondement ontologique de la société. De plus, à l'état naturel, chaque individu est mû par ses passions. Pour Thomas Hobbes, la passion du pouvoir est la principale passion et toutes les autres y sont liées. L'individu, animé par un désir perpétuel d'acquérir de la richesse et du pouvoir, se 
retrouve donc constamment en concurrence avec les autres qui eux aussi désirent plus de richesses et de pouvoir. Vraisemblablement, pour Hobbes, à l'état naturel, chaque individu est en lutte contre les autres, chacun est un loup pour l'autre; d'où la nécessité de fonder l'État. Spinoza pour sa part refuse de tout ramener à la passion du pouvoir et affirme que certains individus peuvent, à l'état naturel, être sages. John Locke, en fervent chrétien, introduit la loi de la nature selon laquelle chacun doit non seulement protéger sa vie, mais aussi celle des autres.

${ }^{28} \mathrm{M}^{\mathrm{gr}}$ Paul Bruchési, "Sur la justice », tome 15, p. 365.

${ }^{29}$ Roy, Progrès, harmonie, liberté, p. 52.

${ }^{30} \mathrm{M}^{\mathrm{gr}}$ Paul Bruchési, «Sur l'affaiblissement de l'esprit chrétien... », tome 13, p. 446.

${ }^{31}$ William Edgar, La carte protestante. Les réformes francophones et l'essor de la modernité (1815-1848), Genève, Labor et Fides, 1997, p. 36.

${ }^{32}$ Ibid., p. 36.

${ }^{33}$ Cet individualisme moderne parait d'abord trouver ses origines lointaines dans la démarche théologique de la Réforme. Selon la théorie de Max Weber dans L'éthique protestante et l'esprit du capitalisme, le protestantisme, en adoptant une perspective plus « individualiste », prend acte d'une mutation religieuse qui, à long terme, encourage l'initiative individuelle et la propriété privée. L'individualisme en tant que tel est une conception qui surgit en Angleterre et en France au siècle des Lumières et même dès le XVII ${ }^{\mathrm{e}}$ siècle avec la parution de Leviathan. Mais il faut attendre Montesquieu pour que soit formulée une véritable doctrine moderne de l'individualisme. Dans De l'esprit des lois, Montesquieu reconnait le caractère inévitable de l'autonomie de l'être humain et il s'efforce de mettre en évidence l'esprit des lois, c'est-à-dire les " rapports nécessaires qui dérivent de la nature des choses ». Selon cette conception, la loi fondée sur la raison humaine perd tout son caractère transcendant. Au XIX ${ }^{\mathrm{c}}$ siècle, Alexis de Tocqueville exprime clairement l'idée d'une évolution historique au cours de laquelle la liberté humaine acquiert un caractère inéluctable. Il reconnaît de même la prépondérance de l'idée de démocratie et de l'individu à la conscience autonome comme préalable à tout ordre politique.

${ }^{34} \mathrm{Il}$ est d'ailleurs intéressant de noter comment la psychologie de l'individualisme moderne semble justement puiser ses racines dans une conception traditionnelle de la conscience qui accorde à l'individu la capacité de choisir et 
de juger par lui-même ce qui est bon pour sa personne, mais qui découle d'une vision essentiellement théocentrique de la personne humaine.

${ }^{35} \mathrm{M}^{\mathrm{gr}}$ Paul Bruchési, «Sur l’affaiblissement de l'esprit chrétien... », tome 13, Montréal, p. 445.

${ }^{36}$ Ibid., p. 446.

${ }^{37}$ Ibid., p. 447-448.

${ }^{38}$ Ibid., p. 448.

${ }^{39}$ Ibid., p. 461.

${ }^{40} \mathrm{Le}$ « Mal » est également dénoncé, dans la correspondance de l'archevêque, sous le nom de "poison".

${ }^{41}$ Les valeurs tels l'honneur, le sacrifice, la tempérance, l'humilité, l'obéissance et le renoncement reflètent le contrôle des passions et assurent la prépondérance de la raison et de la dignité humaine.

${ }^{42}$ Les qualificatifs de « corrompus » ou de « méchants » reviennent souvent dans la correspondance de $\mathrm{M}^{\mathrm{gt}}$ Bruchési. Ils désignent les gens qui se sont détournés de la voie du salut et qui se font les promoteurs du Mal. 